\title{
Tackling the Problem of Overweight and Obesity: The Dutch Approach
}

\author{
Carry M. Renders ${ }^{a, b} \quad J^{b}$ tka Halberstadt ${ }^{b, c}$ Carolien S. Frenkel ${ }^{d} \quad$ Paul Rosenmöller $^{d}$ \\ Jacob C. Seidell ${ }^{\mathrm{a}, \mathrm{b}, \mathrm{c}}$ Remy A. Hirasing ${ }^{\mathrm{a}}$ \\ ${ }^{a}$ Knowledge Centre Overweight, EMGO Institute for Health and Care Research, VU University Medical Centre, \\ ${ }^{\mathrm{b}}$ Faculty of Earth and Life Sciences, Department of Health Sciences, Section Prevention and Public Health, VU University, \\ ' Partnership Overweight Netherlands, EMGO Institute for Health and Care Research, VU University Medical Centre, Amsterdam, \\ ${ }^{\mathrm{d}}$ Covenant on Overweight, The Hague, the Netherlands
}

\section{Keywords}

Obesity · Practical example · Prevention .

Integral approach . Chronic disease management .

National health policy

\section{Summary}

Objective: The aim of this article is to share the Dutch experience of a nation-wide approach to reduce the prevalence of overweight and obesity. This is an practice example of national health policy. Methods: The Ministry of Health facilitates three complementary initiatives to tackle overweight and obesity: i) the Knowledge Centre Overweight (KCO) (since 2002) to enhance knowledge about prevention and treatment of overweight, ii) the Covenant on Overweight (CO) (since 2005), a public-private partnership, iii) the Partnership Overweight Netherlands (PON) (since 2008), to facilitate the development and implementation of a chronic disease management model. Results: The KCO has a platform with 20 partners that functions as a sounding board. It consists of specialists in the area of overweight and obesity. Moreover KCO has a well-visited website: www.overgewicht.org. The $\mathrm{CO}$ with 20 partners from the (local) government, private and public sector has instigated various activities regarding the settings home, school, work, and recreation. The PON has the commitment of 18 partners (organisations of health care providers, health insurance companies and patient organisations) and facilitates the implementation of the national clinical guideline for the diagnosis and treatment of obesity. Conclusion: In the Netherlands relevant stakeholders work together at all levels. The ambition is to make this the first integrated, practice- and evidence-based, national approach for tackling overweight and obesity.

\section{KARGER}

Fax +497614520714

Information@Karger.de

www.karger.com (c) 2010 S. Karger GmbH, Freiburg

Accessible online at:

www.karger.com/ofa

\section{Introduction}

In the Netherlands overweight and obesity have reached epidemic proportions. The prevalence of overweight including obesity among men has increased from $37 \%$ in 1981 to $51 \%$ in 2009 and among women from 30 to $42 \%[1,2]$. In children these prevalences have risen between 1980 and 2003 from 4 to $15 \%$ in boys and from 7 to $18 \%$ in girls [3]. The desire to tackle the problem of overweight and obesity is getting a more prominent place on the Dutch public health as well as the political agenda. In addition, an increasing number and variety of professionals of different public and private partners has become involved in initiatives to prevent or treat overweight and obesity. The Dutch Ministry of Health facilitates three complementary initiatives to tackle the problem: a knowledge centre that focuses on acquiring and disseminating expertise, a covenant that focuses on universal and selective prevention, and a partnership that focuses on indicated/targeted- and care-related prevention.

The Dutch approach to integrate chronic disease management and preventative interventions, may serve as an interesting model for other countries as nation-wide approaches to put knowledge on healthy prevention strategies into practice are missing. In this article the development of this Dutch approach with the three complementary initiatives as well as their objectives and most important activities are described.

\section{Knowledge Centre Overweight (KCO)}

At the end of the 1990s the extent and severity of overweight and obesity in the Dutch population became clear with the results of the 3th (1980) and 4th (1997) national Dutch Growth 
Study in children. Between 1980 and 1997 the prevalence of overweight in children aged 5-12 years more than doubled and that of obesity increased by a factor 8 [3]. This trend was also observed by Youth Health Care (YHC) professionals who asked for tools to intervene in daily practice.

In the Netherlands almost every child in the age of 0-19 years (attendance rate $95 \%$ ) is monitored by a nation-wide program at set ages. This programme is imposed by the government. During the YHC check-ups the growth of each child is measured. YHC professionals not only observed an increasing number of children with overweight but also noticed an increasing degree of overweight as a lot of children did not longer fit into the national growth curves of 1997.

The increasing prevalence in children and also in adults led to an increasing need for knowledge on overweight and obesity and how to deal with it. Therefore, the KCO was established in 2002.

\section{Objectives}

The KCO was established to enhance knowledge about the aetiology, prevention, treatment options, and consequences of overweight and obesity. Its goal is to provide professionals with access to this knowledge and to encourage research. In addition to being a centre for collecting information, the $\mathrm{KCO}$ is a place where prevention officers and care providers can find answers to their questions as well as being an information desk for enquiries and information from, for example the media. Moreover the KCO supports the Ministry of Health, public health services, or local governments in the development of policy in the field of obesity.

In addition, linked to the $\mathrm{KCO}$, a platform was established that functions as a sounding board. It consists of specialists in the area of overweight and obesity in the broadest sense (health promotion organisations, universities and research institutes, representatives of professions of health care providers). It meets several times a year with a view to: informing one another about new developments and projects; coordinating activities; furthering collaboration; upon request providing recommendations about activities, research and interventions in the field of overweight and obesity. The main aim is to achieve optimal coordination between all organisations. As a result, the $\mathrm{KCO}$ has established a wide-ranging network in the field of the prevention and treatment of overweight and obesity.

\section{Most Important Activities}

The KCO has four main activities: to collect and spread knowledge, to stimulate tuning and collaboration between different health supporting organisations, professionals and research to support national and local policy and to generate knowledge.
Website (www.overgewicht.org)

At the start of the $\mathrm{KCO}$, a website was launched to spread the information collected. Since it has been set up, the number of hits has increased enormously from about 500 to more than 6,000 hits/month. The website has proved to be an important tool not only to spread information but also to distribute products of the KCO such as protocols and supporting materials for professionals.

$\mathrm{KCO}$ provides professionals and media with important key publications and state of the art in the field of the prevention and treatment of obesity. Moreover, it supports tuning of projects and activities to prevent duplication of projects and to look to it that financial resources can be applied efficiently. In 2008 the website has undergone a facelift. The main pages have been translated into English.

\section{Youth Health Care (YHC)}

As a response on the increasing requests of YHC doctors for tools to help them deal with the increasing number of overweight children, the KCO developed, in collaboration with the Ministry of Health, a Masterplan to tackle overweight within YHC. YHC was chosen as it is a unique setting to retain or promote a healthy growth, development and behaviour in children, to detect problems, to intervene, and to monitor. As YHC sees almost all children on individual check-ups at set ages, it gives a great opportunity to offer tailored prevention. Moreover, early detection and prevention fits in its mission and in its basic tasks. The Masterplan consisted of different parts:

- The development of an easy tool to determine the BMI in children. YHC doctors indicated that it was not applicable to calculate the BMI of all children in daily practice because most of them were not computerised. Therefore, the BMI nomogram was developed, an easy tool to assess BMI using a lineal.

- A detection protocol developed in 2004 to improve a uniform detection of overweight and obesity in children. It has been distributed by the website of KCO and is in the meantime used in nearly all YHC organisations. The protocol is based on the sex- and age-specific BMI criteria [4]. For borderline cases it is recommended to use clinical vision based on ethnicity, puberty, fat distribution, and external conformation.

- A transitional plan that will be used until an evidencebased programme applicable within daily practice will be available [5]. To develop this intervention a special method was used consisting of 4 activities: i) a literature search to find promising elements to prevent overweight in children and adolescents, ii) consultation of experts in the field of overweight and YHC, iii) an expert meeting among professionals within YHC to make the programme applicable within daily practice and to create commitment, and finally iv) a pilot study.

Five elements were found that are promising in the prevention of overweight in children and adolescents according to 
the scientific literature: i) stimulating breastfeeding, ii) stimulating having breakfast regularly, iii) stimulating physical activity especially playing outside, iv) reducing the consumption of soft drinks and other sweetened drinks, v) reducing watching television and time spent on the computer. All these elements also have other positive side-effects. The transitional plan is published in a book and in various publications within the field of YHC. The transitional plan has received a lot of publicity as it was the first concrete plan to tackle overweight in children that could be structurally implemented. Many YHC organisations are working with this plan. Referring to the transitional plan, also a book with concrete advices and examples for parents was developed. All these products can be downloaded for free from the website of the KCO. This aimed at especially reaching also the socially deprived population. Moreover, the transitional plan is currently evaluated on effectiveness in a randomised clinical trial [6].

The experiences from the YHC can be used by other professions to develop a method to deal with overweight in daily practice. For example on request of the general practitioners the KCO developed a web application to detect and subsequently treat children with overweight and obesity for use in general practice. These tools are based on the tools developed for YHC.

\section{Expert Meetings and Post-Graduate Educations}

In addition the $\mathrm{KCO}$ has organised expert meetings within professions and between different professions aimed at exchanging information to improve collaboration and tuning. This for example has lead to a better tuning of need and demand from YHC and health promoting organisations. Moreover the KCO is involved in post-graduate educations for among others YHC professionals, general practitioners, and dieticians.

\section{Knowledge Generation}

Finally, if knowledge on important subjects is missing or insufficient as noticed by the partners in the platform or from questions from various professionals, KCO will try to improve and fill in this knowledge, e.g. by sharing this knowledge gaps with the research institutes, universities, and other organisations participating in the platform and stimulating tuning and collaboration in research to fill in this gap. Thus it was observed that the awareness of the Dutch population had to be improved to make prevention programmes more effective. Moreover, it was noticed that, in addition to target individual behaviour (demand), more attention should be paid to the environment (supply) to reduce the prevalence of overweight and obesity.

\section{Covenant on Overweight (CO) (Changed in Covenant on Healthy Weight in 2010)}

Consequently in 2004 the Dutch Government announced in her policy document 'Living healthy longer 2004-2007' [7] that citizens are at first responsible for their (un)healthy behaviour, but that healthy behaviour is not always dependent of their doings. The environment also plays a significant role. Therefore, it is important that relevant parties such as food industry, water companies, catering organisations, vending machine suppliers and operators, but also sport confederations, trade unions, local governments and health insurance companies take their responsibility. To make it possible to appeal these parties more on their social responsibility and stimulate self-regulation and tuning, the $\mathrm{CO}$ was initiated in 2005. A well known Dutch former politician was asked to boost the activities of the parties involved.

\section{Objective}

The CO is a public-private partnership, with 20 partners from the (local) government, private sector, and other organisations (like the federations of employers and employees and the Dutch Consumers Association). They work on a joint action plan 'Striking the right balance' (see also $w w w$. convenantgezondgewicht.nl). The partners of the CO apply themselves to make the healthy choice easier for everyone, always at all places. Therefore, the $\mathrm{CO}$ partners and their members are carrying out various activities that can be broadly divided into five key areas: 'Home', 'School', 'Work', 'Recreation' and 'Knowledge and Information'

\section{Most Important Activities}

\section{School}

School is an important place in the lives of children and youngsters, so this is an ideal place to reach them. Therefore, the CO partners are strongly focused on organising activities in and around school. It is also critical to integrate health topics into the curriculum. Activities supported by the $\mathrm{CO}$ at school are diverse.

One of these activities is the Healthy School Cafeteria project which is supported by the Nutritional Centre's Healthy School Cafeteria prize. The goal was to find 75 schools that would provide healthier choices in their cafeterias during the 2008-2009 school year.

In another project in cooperation with water utility companies, the WaterWijs Foundation placed specially designed water coolers TENQs - in secondary schools and regional training centres, so that students can fill up their water bottles for a nominal fee (10 cents). In 2008, the WaterWijs Foundation also introduced TENQY, a smaller version of the TENQ, which is intended for primary schools.

CO partners also support activities to promote healthy nutrition and physical activity amongst primary school pupils by offering exercise classes and healthy lifestyle lessons. Moreover, lessons about food and flavours are given in addition to 
the 'Schoolgruiten' programme that provides primary school pupils with free fruits twice a week, which the children eat as a group.

\section{Home}

Within the setting of home life the $\mathrm{CO}$ and its partners have among others developed the 'I Choose Consciously' logo. This logo can be found on many products by different brands. As a consequence in order to comply with the logo's criteria, many products changed their ingredients.

\section{Work}

As people spend a lot of their time at work, employers and employees can make a significant contribution to the battle against obesity. The $\mathrm{CO}$ and its partners have initiated e.g. the 'Fruit at Work' project. This project aims at increasing the availability of fruits and vegetables in the workplace, for example by placing fruit bowls at prominent locations in organisations. Employees can enjoy the fruit at no cost or for a nominal fee. Moreover, the $\mathrm{CO}$ and partners stimulate companies in having an exercise policy and more employees engaging in an active lifestyle (at least $70 \%$ ).

\section{Recreation}

Recreational and leisure activities are things you do because you enjoy them. The $\mathrm{CO}$ is offering opportunities to unwind through sport and is striving to make recreational environments as healthy as possible. Therefore, the CO supports projects such as developing healthy playgrounds, a campaign to increase the number of people in the catering industry who use liquid cooking fats (instead of solid fats) and the project 'Score for Health'. This last project takes advantage of the popularity of professional soccer players. Dutch Premiership Division players provide children with a healthy example over the course of 20 weeks. In addition, 'Score for Health' features a topic related to physical activity and healthy nutrition every 3 weeks.

\section{Partnership Overweight Netherlands (PON)}

As a consequence of the increasing attention from various professionals to take part in the prevention and treatment of overweight and obesity and the need for the effort to connect the preventive activities with treatment, the PON was initiated by the Dutch Ministry of Health in 2008. The PON is a collaboration between 18 partners, which are national organisations of health care providers, health insurance companies, and patient organisations.

\section{Objective}

Objective of the PON is to facilitate the development and implementation of a chronic disease management model for overweight and obesity. This model involves strategies for diagnosis and early detection of high-risk individuals as well as appropriate integrated lifestyle interventions for those who are overweight and obese and, when appropriate, additional medical therapies.

The PON works towards an integrated health care system that transcends traditional boundaries of conventional health care systems and health care professions but, instead, focuses on competences of groups of health professionals who organise care from a patient oriented perspective.

The PON collaborates with organisations responsible for prevention and management of other chronic diseases such as type 2 diabetes and cardiovascular diseases, and thus also transgresses the boundaries of chronic disease specific health care systems. The systems are following the organisation of health care as described by Wagner et al. [9] and Bodemheimer [9] as the Chronic Care Model.

The Chronic Care Model is based on the use of evidence based, planned care; re-organisation of practice systems and provider roles; improved patient self-management support; increased access to expertise; and greater availability of clinical information. The challenge is to organise these components into an integrated system of chronic illness care.

\section{Most Important Activities}

\section{Guideline Implementation}

The PON is developing flow charts for different age groups and different degrees of overweight and obesity to facilitate the implementation of the national 'Clinical Guideline for the Diagnosis and Treatment of Obesity in Adults and Children' with implications for the organisation, quality assurance, and reimbursement of care and prevention [10].

These flow charts are described for different levels of weight related health risk which, for adults, is based on BMI, waist circumference, and co-morbidity. For children and adolescents it is based on BMI and co-morbidity. Figure 1 illustrates the pyramid of overweight and obesity and the levels of risk and intensity of interventions.

\section{Method of Working}

All activities of the PON are supported by a management team and a small staff. Yearly four meetings with representatives of the 18 partners take place. All partners have signed a declaration of intent to show their commitment to the shared goals.

Multidisciplinary working groups are formed each year to work on answering diverse questions and on distinctive aspects of the flow charts. The results of the working groups and the meetings of the partners are summarised during a yearly conference. The conference is also a starting point for the activities in the following year. 


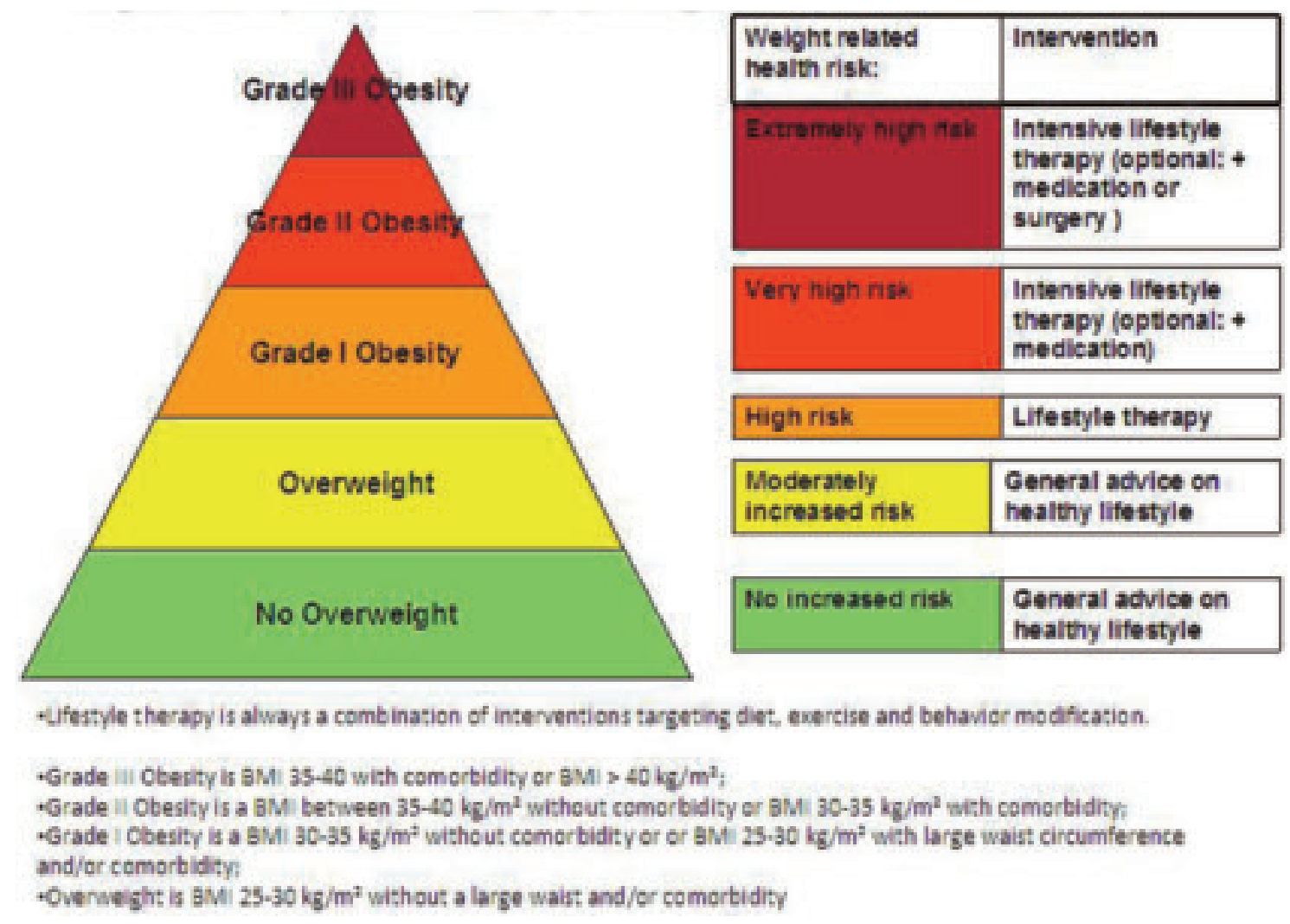

Fig. 1. Pyramid obesity management in adults.

only accessible for the representatives of the partner organisations and the members of the working groups. This section features working documents that are not ready to publish yet. health care providers are represented: general practitioners, dieticians, physical therapists, psychologists, doctors and nurses in youth health care, paediatricians, doctors in internal medicine, physicians in occupational health, pharmacists, nurses, and surgeons.

The Dutch health insurance companies are represented by their national organisation, and the patients are represented by the Dutch Obesity Association and the more general federation for patient and consumer organisations.

The participants in the working groups of the PON consist of representatives of the 18 partner organisations and of relevant experts of organisations that are not a partner of the PON. These include for example experts in statistics, medical ethics, and self management. The PON also has close ties with the Dutch platform that is responsible for the coordination of the development of chronic disease management models for related disorders such as obesity, type 2 diabetes, and cardiovascular diseases.

Furthermore, the PON has close collaboration and knowledge exchange with different departments of the Ministry of Health.

\section{Website}

The PON has a website (www.partnerschapovergewicht.nl) which functions as a means of communicating information regarding its activities. The website also has a section that is

\section{Future/Conclusion}

In the Netherlands an important step is taken in tackling overweight and obesity with establishing the three described complementary initiatives. Firstly, the KCO was initiated to acquiring and disseminating expertise about prevention and treatment of overweight. Moreover, it stimulates tuning and exchange of information between policy, research, health promoting institutes, and professionals. Secondly, the $\mathrm{CO}$ was established to encourage cooperation between public and private parties from diverse segments of society with special attention for changing the environment. Finally, the PON was initiated to facilitate the development and implementation of a chronic disease management model for overweight and obesity.

The $\mathrm{KCO}, \mathrm{CO}$, and PON regularly publish about their activities including press releases, brochures, and articles in specialist journals. Additionally, they are regularly interviewed and give oral presentations at (inter)national meetings and conferences.

The collaboration of these three complementary initiatives shows that stakeholders relevant to the prevention and management of overweight and obesity are willing and able to 
work together at all levels. The ambition is to make this the first integrated, evidence based and practice based, national approach for tackling the problem of overweight and obesity.

Tackling obesity has to be part of not only national but also local health policy. Therefore, a start is made to link the national activities to local demonstration projects. These projects aim to monitor and evaluate community intervention approaches based on the EPODE initiative to prevent overweight and obesity [11]. These programmes target schools, home environments, and neighbourhoods. Other projects aim to integrate prevention and management of overweight and obese individuals and those who are high at risk.

Although the integrated national approach seems to be broadly influencing the entire population because of the networking of different actions on various levels and from different settings, the impact and power of this approach has to be proved in the future. Therefore we also work on monitoring systems for the prevalences of overweight and obesity. YHC gives a good opportunity to monitor trends in prevalences of overweight and obesity on a more regular basis and is working on an uniform digital record system.

Nevertheless, in the Netherlands we succeeded in bringing all relevant stakeholders together that can contribute to tackle the problem of overweight and obesity. Now we should focus on an efficient execution and evaluation in which parties involved collaborate and tune together, on the national and local level. This is supported by the Ministry of Health and Ministry of Youth and Family as described in the policy document: 'Overweight: Out of Balance the Burden of Overweight' [12]. Finally a blue print to support local governments, public and private partners and professionals to integrate chronic disease management and preventative interventions should be developed.

The staff of the 3 initiatives consists per initiative of one project manager, 1-4 project assistants, one secretary and a Board.

\section{Acknowledgements}

The three complementary initiatives are funded by the Dutch Ministry of Health. We thank the Ministry of Health for their support.

\section{Disclosure}

The authors declared no conflict of interest.

\section{References}

1 Statistics Netherlands (CBS): Heerlen, the Netherlands, 2009.

2 Schokker DF, Visscher TL, Nooyens AC, van Baak MA, Seidell JC: Prevalence of overweight and obesity in the Netherlands. Obes Rev 2007;8:101-108.

3 Van den Hurk K, van Dommelen P, van Buuren S, Verkerk PH, Hirasing RA: Prevalence of overweight and obesity in the Netherlands in 2003 compared to 1980 and 1997. Arch Dis Child 2007; 92:992-995.

4 Cole TJ, Bellizzi MC, Flegal KM, Dietz WH: Establishing a standard definition for child overweight and obesity worldwide: international survey. BMJ 2000;320:1240-1243.

5 Bulk-Bunschoten A, Renders CM, Hirasing RA Noodplan voor te zware kinderen. Medisch Contact 2006;61:1206-1209.
6 Veldhuis L, Struijk MK, Kroeze W, Oenema A, Renders CM, Bulk-Bunschoten AMW, HiraSing $\mathrm{RA}$, Raat H: 'Be active, eat right', evaluation of an overweight prevention protocol among 5-year-old children: design of a cluster randomised controlled trial. BMC Public Health 2009;9:177

7 Policy document: 'Living healthy longer 2004-2007'. www.rijksoverheid.nl/documenten-en-publicaties/ notas/2009/03/23/nota-overgewicht.html.

8 Wagner EH, Austin BT, Von Korff M: Organizing care for patients with chronic illness. Millbank Quarterly 1996;74:511-544.

9 Bodemheimer T: Interventions to improve chronic illness care: evaluating their effectiveness. Dis Management 2003;6:63-71.
10 Kwaliteitsinstituut voor de Gezondheidszorg CBO Clinical guideline for the diagnosis and treatment of obesity in adults and children (in Dutch). Van Zuiden Communications BV, Alphen aan den Rijn, 2008.

11 Romon M, Lommez A, Tafflet M, Basdevant A Oppert JM, Bresson JL Ducimetière P, Charles MA, Borys JM: Downward trends in the prevalence of childhood overweight in the setting of 12-year school- and community-based programmes. Public Health Nutr 2009;23:1-8.

12 Policy document: 'Overweight: Out of Balance the Burden of Overweight' (in Dutc)hMinistry of Health and Ministry of Youth and Family, Den Haag, 2009. 